\title{
SPECTRAL ANALYSIS OF THE STRUCTURE OF ULTRADISPERSED DIAMONDS
}

\author{
V. V. Uglov, ${ }^{a *}$ V. I. Shimanski, ${ }^{a}$ D. P. Rusalsky, ${ }^{a}$ \\ and M. P. Samtsov
}

UDC 543.422:548.736.15

\begin{abstract}
The structure of ultradispersed diamonds (UDD) is studied by spectral methods. The presence of diamond crystal phase in the UDD is found based on x-ray analysis and Raman spectra. The Raman spectra also show $s p^{2}$ - and $s p^{3}$-hybridized carbon. Analysis of IR absorption spectra suggests that the composition of functional groups present in the particles changes during the treatment.
\end{abstract}

Key words: ultradispersed diamonds, x-ray analysis, Raman scattering, infrared spectroscopy.

Introduction. The detonation of explosives in closed chambers can produce ultradispersed carbon powders that are of practical interest because of the properties of the particles formed. The detonation of solid explosives creates conditions (20-30 GPa, 3000-4000 K) that promote the formation of diamond particles. Therefore, the synthesized powders contain a large amount of ultradispersed diamonds (UDD). As a rule, the produced diamonds are spherical with a diameter of about $4 \mathrm{~nm}[1,2]$. UDD of such small dimensions are used in industry as an additive for fabricating composites [3], as catalysts for carrying out certain chemical reactions [4], etc. A detailed investigation of the properties and structure of UDD particles could expand their applications. Being small particles with a high surface energy, UDD are stabilized as blocks (clusters) $20-30 \mathrm{~nm}$ in size. This hinders the investigation of the structure of the individual particles and requires the use of methods that can provide information for rather bulky samples.

A certain aspect of UDD structure has been described in most work related to the investigation of their structure. This is either the crystal structure or the presence of functional groups in them. The goal of our work was to investigate comprehensively UDD particles using various spectral methods and to find the functional groups that appear during chemical isolation of UDD from the detonation charge.

Experimental. Samples of UDD were obtained from products of detonation synthesis (detonation charge) through explosion of carbon-containing compounds such as trinitrotoluene and hexogen. The parameters of the medium in the detonation wave (pressure and temperature) corresponded to the formation of diamond. The UDD were obtained by barometric oxidation of the detonation charge diluted with nitric acid. After the reaction was finished, samples were extracted from the acidic solution and washed in distilled water. The samples were sent to NP ZAO "Sinta" (Minsk).

The phases and structure of UDD were studied using x-ray diffraction analysis on a DRON-3 x-ray diffractometer with monochromatic copper radiation of wavelength $1.54178 \mathrm{~nm}$. Powder spectra of UDD on a glass substrate were recorded in the range of angles $20-120^{\circ}$.

Samples were prepared as disks pressed from a mixture of UDD powder and $\mathrm{KBr}(1 \%$ mass content of UDD powder). Raman spectra were recorded at room temperature using a Ramalog 4 spectrometer (Spex) with excitation by $\mathrm{Ar}^{+}$-laser radiation $(488$ or $514 \mathrm{~nm}$ ) with power on the sample of $0.10-0.15 \mathrm{~W}$ and beam diameter $\sim 10 \mu \mathrm{m}$. IR absorption spectra in the range $4000-500 \mathrm{~cm}^{-1}$ were measured using a single-beam Fourier Vertex 70 spectrometer (Bruker) with resolution $0.5 \mathrm{~cm}^{-1}$ with averaging over 20 scans.

Results and Discussion. Figure 1 shows x-ray diffraction patterns from samples of detonation charge and UDD. A feature of the resulting patterns at small reflection angles is a steeply increasing background, the presence of which is due to scattering from amorphous dispersed structures. Also, a relatively high intensity background over the

${ }^{*}$ To whom correspondence should be addressed.

${ }^{\mathrm{a}}$ Belarusian State University, 4 Nezavisimosti Ave., Minsk, 220030, Belarus; e-mail: Uglov@bsu.by; ${ }^{\mathrm{b}}$ A. N. Sevchenko Institute of Applied Physics Problems, Belarusian State University, Minsk. Translated from Zhurnal Prikladnoi Spektroskopii, Vol. 75, No. 4, pp. 524-528, July-August, 2008. Original article submitted November 6, 2007. 


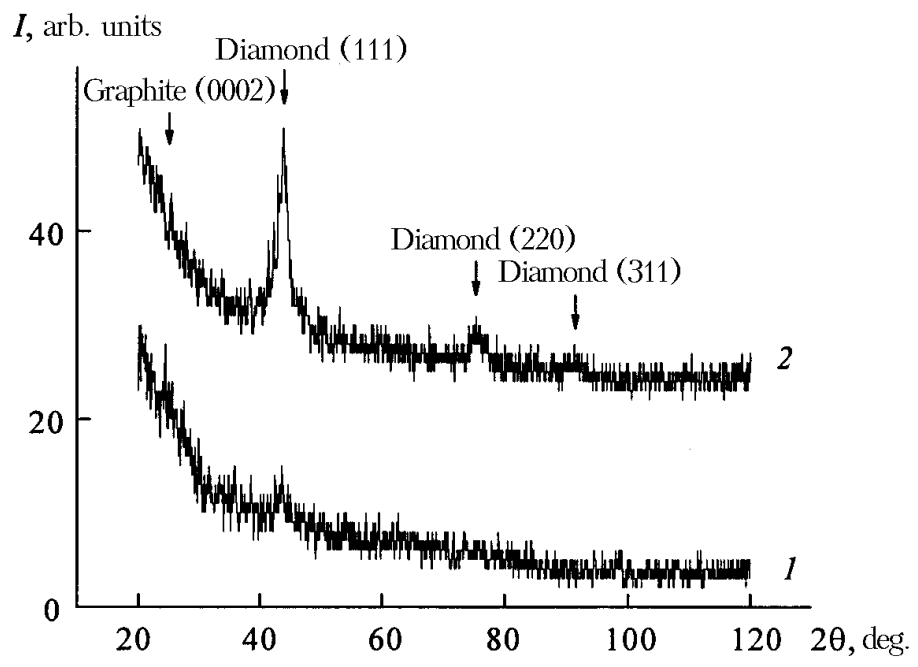

Fig. 1. X-ray diffraction patterns of detonation charge (1) and ultradispersed diamonds (2).

whole range of diffraction angles is found, indicating the presence of a large number of fine amorphous particles in the sample. The pattern of the detonation charge exhibits a weak diffraction maximum at diffraction angle $2 \theta \approx 25^{\circ}$ that can be assigned to reflection from the (0002)-plane of graphite. This maximum is missing in the pattern of UDD. This condition suggests that the graphite formed upon detonation is removed after chemical purification of the detonation charge. The pattern of UDD exhibits broad symmetric maxima at diffraction angles $2 \theta=43.8,75.2$, and $91.6^{\circ}$ that correspond to reflections from diamond planes (111), (220), and (311) [5]. The presence of these diffraction maxima in the pattern of UDD indicates that a significant amount of the crystalline diamond phase of carbon is present in the samples. The intensity of the diffraction line at $2 \theta=43.8^{\circ}$ in the pattern of UDD increases compared with its intensity in the pattern of the charge. Two other lines observed in the pattern of UDD are missing in the pattern of the charge. This is consistent with an increased relative content of the diamond phase in the UDD samples, which is achieved by carrying out the corresponding chemical treatment by which non-diamond particles are removed from the charge. The lattice constant of diamond calculated from the strongest line is $0.357 \mathrm{~nm}$. This value agrees fully with the lattice constant of macroscopic diamond.

The observed diffraction maxima in the pattern of UDD are rather broad (several degrees at half-height). It is known that the fundamental reasons producing broad diffraction lines are microstresses within the sample and its particle size [6]. If existing internal microstresses are ignored and it is assumed that the width of the diffraction lines is due only to the particle size of the diamond powder, then the average particle size of UDD can be estimated from the Debye-Scherrer equation. The average particle size $L$ is determined using the formula $L=\lambda /(d \cos \theta)$, where $d$ is the width of the diffraction line at half-height for diffraction angle $\theta$ and $\lambda$ is the wavelength of the $\mathrm{x}$-ray radiation. The calculated average particle size of UDD is $\sim 4 \mathrm{~nm}$.

The results of the x-ray diffraction study agree with those obtained by Raman scattering. Figure 2 shows Raman spectra of the detonation mixture and UDD. The spectrum of the detonation mixture exhibits only two broad bands with maxima at 1356 and $1592 \mathrm{~cm}^{-1}$ that are due to a high content in the sample of $s p^{2}$ - and $s p^{3}$-hybridized carbon (including graphite) [7, 8]. The spectrum of UDD contains a rather narrow band at $1330 \mathrm{~cm}^{-1}$, the presence of which is due to the crystalline diamond phase of carbon. A second characteristic band for UDD in the Raman spectrum is located at $1088 \mathrm{~cm}^{-1}$. Besides these lines, a broad diffuse band at $1570 \mathrm{~cm}^{-1}$ that can be assigned to vibrations of $s p^{2}$-hybridized bonds in aromatic rings and aliphatic chains is observed. The differences in the Raman spectra of the charge and UDD indicate that the composition of the powder-particle shell was changed by the treatment.

IR absorption spectra of the detonation charge and UDD (Fig. 3) exhibit vibrations of various functional groups, the assignments of which were made based on an analysis of the results and a comparison with known data for such samples [9-12]. The spectrum of the detonation charge contains a broad maximum at $590 \mathrm{~cm}^{-1}$ that is most 


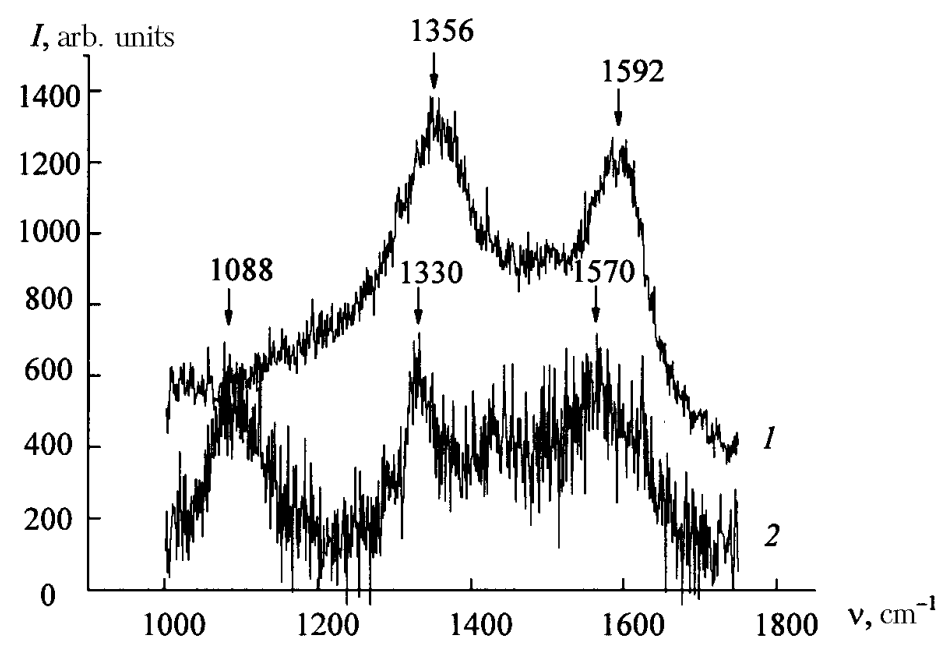

Fig. 2. Raman spectra of detonation charge (1) and ultradispersed diamonds (2).

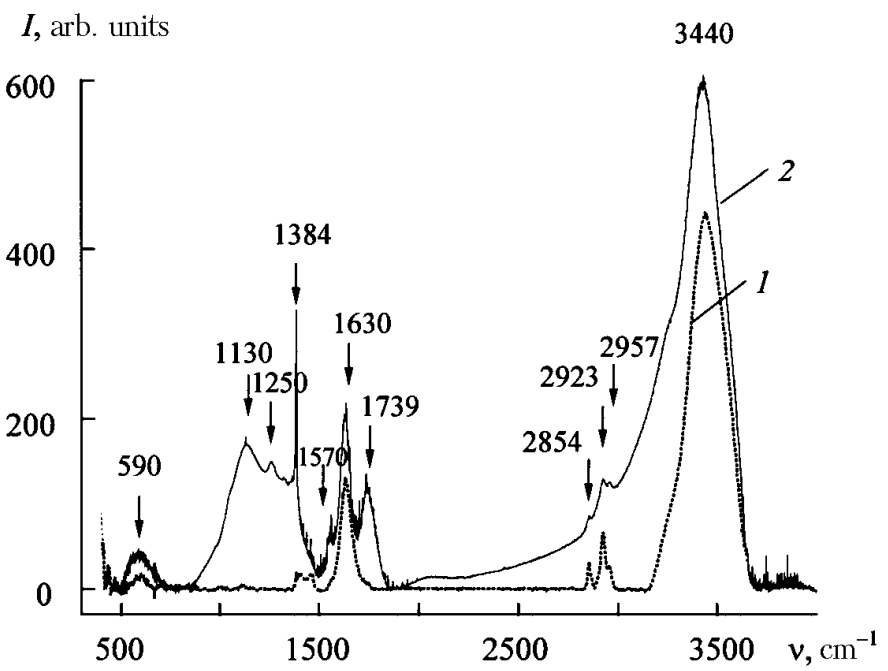

Fig. 3. IR absorption spectra of detonation charge (1) and ultradispersed diamonds (2).

probably due to vibrations of a $\equiv \mathrm{C}-\mathrm{O}-\mathrm{C} \equiv$ group. Two closely spaced bands with maxima at 1384 and $1460 \mathrm{~cm}^{-1}$ belong to symmetric and antisymmetric vibrations of $\mathrm{CH}_{3}$ groups, respectively [13]. Absorption bands at 1630 and 3440 $\mathrm{cm}^{-1}$ are due to deformation and stretching vibrations of $\mathrm{O}-\mathrm{H}$ groups in water molecules bound and adsorbed to UDD particles [12]. The appearance of water absorption bands is typical of all IR spectra of UDD [14]. The presence of a maximum at $3440 \mathrm{~cm}^{-1}$ and its washout at lower wavenumbers indicates, according to the literature [14], that a water monolayer with intermolecular hydrogen bonds is formed. The doublet at 2854 and $2923 \mathrm{~cm}^{-1}$ corresponds to stretching vibrations of $s p^{3}$-hybridized $\mathrm{CH}_{3}$.

Additional absorption maxima besides the examined bands appeared in the absorption spectrum of UDD after processing of the charge. A broad band with maximum at $1130 \mathrm{~cm}^{-1}$ was probably due to absorption of nitrogen impurities. The presence of nitrogenous molecules in the UDD samples is explained by the use of nitric acid to isolate UDD from the detonation charge. The rather narrow and strong absorption band at $1384 \mathrm{~cm}^{-1}$ corresponds to $\mathrm{CH}_{3}$ vibrations. It should be noted that this band in analogous spectra [15] is practically not observed, like the band at 1570 $\mathrm{cm}^{-1}$, which can be correlated with symmetric vibrations of $\mathrm{NO}_{2}$ [13]. The absorption band with maximum at 1739 $\mathrm{cm}^{-1}$ is due to stretching vibrations of the $\mathrm{C}=\mathrm{O}$ functional group; at $2957 \mathrm{~cm}^{-1}$, to stretching vibrations of $s p^{3}$-hybridized $\mathrm{CH}_{2}$. 
Thus, samples containing UDD typically have a significant number of functional groups, many of which are missing in the detonation charge. The observed differences in the spectra during chemical processing are due to surface modification of the particles. A similar picture is characteristic for finely dispersed diamond materials [9]. Many studies $[4,15]$ have shown that the surface of synthetic diamond powder is coated with oxygen-containing functional groups, the concentration of which is greater, the greater the active surface area. Various organic surfactants also adsorb well to the surface of diamond. As a result, surface functional groups contribute substantially to the IR absorption spectra of diamond powders.

According to the core theory [4] particles of nanodiamond are supermolecules with a single-crystalline diamond nucleus (core) that is surrounded by a chemical coating of functional groups bound to it that determine the condition of the UDD surface. Thus, for UDD particles with an average diameter of $\sim 4 \mathrm{~nm}$, the fraction of surface atoms is $\sim 15 \%$. Therefore, the symmetry of carbon-atom positions is destroyed and the lattice constant is decreased. This results in the properties of UDD being determined to a large extent by the condition of their surface.

Conclusion. X-ray diffraction established the structure and phase composition of particles of UDD. These are nano-sized particles with the diamond crystal lattice, the dimensions of which are $\sim 4 \mathrm{~nm}$. Raman and IR spectroscopy revealed the influence of purification conditions of the UDD on the chemical composition of their surface. The crystal lattice is most probably surrounded by an amorphous coating of hydrocarbon, carboxylic, and nitrogen-containing functional groups that apparently make up a significant part of the UDD particles.

Acknowledgments. We thank A. P. Korzhenevskii and R. G. Shtemplyuk for supplying samples and providing valuable comments about the article.

\section{REFERENCES}

1. V. D. Andreev, and Yu. I. Sozin, Fiz. Tverd. Tela, 41, No. 10, 1890-1893 (1999).

2. A. E. Aleksenskii, M. V. Baidakova, A. Ya. Vul', and V. I. Siklitskii, Fiz. Tverd. Tela, 41, No. 4, 740-743 (1999).

3. G. K. Burkat and V. Yu. Dolmatov, Fiz. Tverd. Tela, 46, No. 4, 685-692 (2004).

4. I. I. Kulakova, Fiz. Tverd. Tela, 46, No. 4, 621-628 (2004).

5. Powder Diffraction File, JCPDS, International Centre for Diffraction Data, American Society for Testing and Materials (ATSM), USA, No. 6-0675 (1977).

6. A. A. Rusakov, X-ray Diffraction of Metals [in Russian], Atomizdat, Moscow (1977).

7. A. C. Ferrari and J. Robertson, Phys. Rev. B: Condens. Matter Mater. Phys., 61, No. 20, 14095-14107 (2000).

8. A. C. Ferrari and J. Robertson, Phys. Rev. B: Condens. Matter Mater. Phys., 63, 121405-1-121405-4 (2001).

9. N. V. Novikov, D. V. Fedoseev, A. A. Shul'zhenko, and G. P. Bogatyrev, Diamond Synthesis [in Russian], Naukova Dumka, Kiev (1987).

10. D. S. Roberts, Ind. Diamant. Rdsch., 13, No. 2, 144-148 (1978).

11. V. G. Aleshin, G. P. Bogatyrev, I. K. Kruk, and A. A. Smekhnov, Dokl. Akad. Nauk Ukr. SSR, Ser. A, No. 3, 75-77 (1985).

12. G. V. Sakovich, V. F. Komarov, and E. A. Petrov, Sverkhtverd. Mater., No. 3, 3-6 (2002).

13. A. I. Komyak, Molecular Spectroscopy [in Russian], Bel. Gos. Univ., Minsk (2005).

14. J. Shengfu, T. Jiang, K. Xu, and S. Li, Appl. Surf. Sci., 133, 231-238 (1998).

15. E. Mironov, A. Koretz, and E. Petrov, Diamond Relat. Mater., 11, $872-876$ (2002). 